\title{
Harmonic Analysis and Simulation Study Using Triple-spectrum-line interpolation FFT Algorithm
}

\author{
Xiao-wan SONG ${ }^{1, a}$, De-min $\mathrm{LI}^{2, \mathrm{~b}}$, Zhen-hua $\mathrm{LI}^{3, \mathrm{c}}$, Wen-zhang GUO ${ }^{4, \mathrm{~d}}$ \\ ${ }^{1}$ Hubei Key Laboratory of Cascaded Hydropower Stations Operation \& Control, China Three \\ Gorges University, Yichang Hubei 443002, China \\ ${ }^{2}$ School of Electrical Engineering and Automation, Hefei University of Technology, Hefei Anhui \\ 230009, China \\ ${ }^{3}$ Hubei Key Laboratory of Cascaded Hydropower Stations Operation \& Control, China Three \\ Gorges University, Yichang Hubei 443002, China \\ ${ }^{4}$ Hubei Key Laboratory of Cascaded Hydropower Stations Operation \& Control, China Three \\ Gorges University, Yichang Hubei 443002, China \\ axiaowansongst@163.com, b2360534795@qq.com, clizhenhua1993@163.com, \\ dgwz_alive@163.com
}

Keywords: Fast Fourier Transform, Spectral Leakage, Harmonic Analysis, Triple-spectrum-line Interpolation, Harmonic Frequency Point

\begin{abstract}
There is hard to obtain the harmonic parameters exactly for the fast Fourier transform in situation of non-synchronous sampling. The cosine window and triple-spectrum-line interpolation FFT algorithm is presented to reduce spectral leakage and improve the accuracy of harmonic analysis. The windowed FFT transform is applied to the sampling signal using a good sidelobe characteristic window and discrete spectrum lines are obtained. The weighted arithmetic is operated by using three spectrum lines near the harmonic frequency point and the applicable rectifications of triple-spectrum-line FFT are deduced. Simulation results show that the algorithm has a higher accuracy of harmonic analysis compared with the cosine windowed double interpolation FFT in non-synchronized sampling.
\end{abstract}

\section{Introduction}

With the technical development, nonlinear devices such as transformers and power electronic instruments are widely applied in the process of power conversion and transportation, however, harmonic pollution in power system also increases along with it [1]. High accuracy analysis of the harmonic parameters is essential for electric energy measurement, harmonic flow calculation, harmonic compensation and suppression, etc. [2].

Fast Fourier transform (FFT) is the most common method used for harmonic analysis. By adopting the synchronous sampling technology, FFT algorithm can accurately analyze the harmonic amplitude, frequency and phase. However, the frequency of the power network is not a constant, it is difficult to achieve strict synchronous sampling when using the discrete phase locked loop technique [3] or adjusting the sampling frequency by the software [4]. When adopting non-synchronous sampling, FFT algorithm will produce spectrum leakage and fence effect, leading to the calculation of harmonic parameters (frequency, amplitude and phase) inaccurate [5]. Research has shown that FFT algorithm based on the sampling data windowing and interpolation correction for transformation results can effectively reduce the spectrum leakage and improve the accuracy of harmonic analysis. Common window functions are Hanning window [6], Blackman window [7], and Nuttall windows [8], etc. Doublet interpolation method [9] is commonly used as interpolation correction algorithm, but the algorithm does not take fully use of information contained in the leak spectral line of the harmonic frequency point and its nearby.

The spectrum of Blackman-Harris window is analyzed in this paper with its very low sidelobe peak level features, which are used to harmonic signals to reduce spectral leakage effect. Taking full advantage of information contained in the leak spectral line of the harmonic frequency point 
and its nearby, selecting three discrete spectral lines of the harmonic frequency point to interpolate correction, and the correction of the three spectral line interpolation based on Blackman-Harris window is derived. Simulation results show that in non-synchronous sampling, compared with the FFT algorithm, the Blackman window and the Blackman-Harris window double spectrum lines interpolation FFT algorithm, the algorithm proposed in this paper has higher precision in harmonic analysis.

\section{Three Spectral Line Interpolation based on Blackman-Harris Window}

\section{Spectral Characteristics of Blackman-Harris Window}

Adopting window function fixed excellent sidelobe performance to window the signal can reduce spectral leakage effect. Blackman-Harris window is a kind of four items raised-cosine window, its time-domain expression is

$$
w(n)=0.35875-0.48829 \cos \left(\frac{2 \pi n}{N}\right)+0.14128 \cos \left(\frac{4 \pi n}{N}\right)-0.01168 \cos \left(\frac{6 \pi n}{N}\right)
$$

with $n=0,1, \ldots . . N-1$, when $N \gg>1$, DTFT based on Blackman-Harris window is converted into:

$$
\begin{aligned}
& W(w)=0.35875 W_{\mathrm{R}}(w)-0.224145\left[W_{\mathrm{R}}\left(w-\frac{2 \pi}{N}\right)+W_{\mathrm{R}}\left(w+\frac{2 \pi}{N}\right)\right]+ \\
& 0.224145\left[W_{\mathrm{R}}\left(w-\frac{2 \pi}{N}\right)+W_{\mathrm{R}}\left(w+\frac{2 \pi}{N}\right)\right]+0.07064\left[W_{\mathrm{R}}\left(w-\frac{4 \pi}{N}\right)+W_{\mathrm{R}}\left(w+\frac{4 \pi}{N}\right)\right]- \\
& 0.00584\left[W_{\mathrm{R}}\left(w-\frac{6 \pi}{N}\right)+W_{\mathrm{R}}\left(w+\frac{6 \pi}{N}\right)\right]
\end{aligned}
$$

with $W_{\mathrm{R}}(w)=\frac{\sin (w N / 2)}{\sin (w / 2)} e^{-\mathrm{j} \frac{N-1}{2} w}$ is a spectral function of the rectangular window.

As shown in Table 1, sidelobe peak level of Hanning window and Hamming window is relatively high, and side-lobe characteristics of those two window functions are both not ideal. Asymptotic decay rate of sidelobe of Blackman window is faster than that of Blackman-Harris window, but sidelobe peak level of Blackman-Harris window is $-92 \mathrm{~dB}$ (The negative sign indicates sidelobe peak level below the main lobe level), which is far below the $-58 \mathrm{~dB}$ of Blackman window, showing that Blackman-Harris window has good sidelobe characteristics. Thus, in this paper, we choose Blackman-Harris window to process harmonic signal in interpolating windowed FFT algorithm.

Table 1. Sidelobe characteristics of several cosine windows

\begin{tabular}{lcc}
\hline Windows & $\begin{array}{c}\text { Peak of } \\
\text { sidelobe }(\mathrm{dB})\end{array}$ & Rate of attenuation(dB/oct) \\
\hline Hanning & -32 & 18 \\
Hamming & -43 & 6 \\
Blackman & -58 & 18 \\
Blackman-Harris & -92 & 6 \\
\hline
\end{tabular}

\section{Triple-Spectrum-Line Interpolation Algorithm based on Window FFT}

A single frequency signal $x(t)$ is uniformly sampled by a sampling frequency $f_{\mathrm{s}}$, and the discrete time signal is obtained:

$$
x(n)=A_{0} \sin \left(2 \pi n f_{0} / f_{s}+\varphi_{0}\right)
$$

with $A_{0} 、 f_{0} 、 \varphi_{0}$ are the signal amplitude, frequency and initial phase, respectively, $n=0,1, \cdots$, $N-1$. 
$x(n)$ is windowed by Blackman-Harris window and $x_{\mathrm{w}}(n)=x(n) w(n)$ is obtained, with $w(n)$ is the time-domain expression of Blackman-Harris window. Continuous Fourier transform is used for $x_{\mathrm{w}}(n)$ in frequency-domain, and sidelobes effect at frequency peak of the negative frequency point is neglected, then frequency-domain expression of $x_{\mathrm{w}}(n)$ is obtained:

$$
X_{w}(f)=\sum_{0}^{\infty} x(n) w(n) e^{-\mathrm{j} 2 \pi n f}=\frac{A_{0}}{2 j}\left\{e^{\mathrm{j} \varphi_{0}} W\left[\frac{2 \pi\left(f-f_{0}\right)}{f_{s}}\right]\right\}
$$

with $W(f)$ is the spectrum function of continuous frequency of Blackman-Harris window. The discrete sampling is implemented to (4), and the discrete spectrum of $x_{\mathrm{w}}(n)$ is obtained:

$$
X(k \Delta f)=A_{0} e^{\mathrm{j} \varphi_{0}} W\left[\frac{2 \pi\left(k \Delta f-f_{0}\right)}{f_{s}}\right] /(2 j)
$$

with $\Delta f=f_{\mathrm{s}} / N$ is frequency resolution, $N$ is data truncation length, namely the number of sampling points.

When non-synchronized sampling, the sampling points fail to reach harmonic frequency points and thus frequency spectrum lines can't be obtained, $k_{0}=f_{0} / \Delta f$ is the non-integer. Set $k_{\mathrm{p}}$ th spectrum line is the maximum amplitude spectrum line of the neighborhood of spectrum line $k_{0}$ at the harmonic frequency point, the left and right sides of the $k_{\mathrm{p}}$ th spectrum line, are $k_{\mathrm{p}-1}$ th spectrum line and $k_{\mathrm{p}+1}$ th spectrum line $\left(k_{\mathrm{p}}=k_{\mathrm{p}-1}+1, k_{\mathrm{p}+1}=k_{\mathrm{p}}+1\right)$, respectively. The amplitudes of these three lines are $y_{\mathrm{p}-1}=\left|X\left(k_{\mathrm{p}-1} \Delta f\right)\right|, y_{\mathrm{p}}=\left|X\left(k_{\mathrm{p}} \Delta f\right)\right|$ and $y_{\mathrm{p}+1}=\left|X\left(k_{\mathrm{p}+1} \Delta f\right)\right|$, respectively. Parameter $\alpha=k_{0}-k_{\mathrm{p}}(-0.5 \leq \alpha \leq 0.5)$, $\beta=\left(y_{\mathrm{p}+1}-y_{\mathrm{p}-1}\right) / y_{\mathrm{p}}$ are introduced. According to (5),

$$
\beta=\frac{\left|W\left(\frac{2 \pi(-\alpha+1)}{N}\right)\right|-\left|W\left(\frac{2 \pi(-\alpha-1)}{N}\right)\right|}{\left|W\left(\frac{2 \pi(-\alpha)}{N}\right)\right|}
$$

set the (6) as $\beta=f(\alpha)$, whose inverse function is $\alpha=f^{-1}(\beta)$.

Since $k_{\mathrm{p}}$ th spectrum line is the maximum amplitude spectrum line of the neighborhood of spectrum line $k_{0}$ at the harmonic frequency point, greater weight value is given to the line when seeking amplitude correction. Compared with[10] which weighted values of $k_{\mathrm{p}-1}$ th $、 k_{\mathrm{p}}$ th $、 k_{\mathrm{p}+1}$ th line is directly given as $1 、 2$ and 1 , this paper proposals a new method: At first, a single frequency signal $x(n)=\sin \left(2 \pi n f_{0} / f_{\mathrm{s}}\right)$ with the amplitude of 1 is windowed by Blackman-Harris window to conduct FFT analysis, with $f_{0}=50 \mathrm{~Hz}$, n equals 512 points, $f_{\mathrm{s}}=2560 \mathrm{~Hz}$, then find $k_{\mathrm{p}-1}$ th $、 k_{\mathrm{p}}$ th $、 k_{\mathrm{p}+1}$ th spectrum line in the discrete spectrum and its amplitude, however, $k_{\mathrm{p}}$ th spectrum line amplitude $y_{\mathrm{p}}$ is less than 1 due to the spectrum leakage effect. FFT processing results of $y_{\mathrm{p}}$ at this time is 0.3587 , $k_{\mathrm{p}-1}$ th $、 k_{\mathrm{p}+1}$ th spectrum line amplitude are $y_{\mathrm{p}-1}=0.2403 、 y_{\mathrm{p}+1}=0.2480$, respectively. Because other discrete spectral lines except the $k_{\mathrm{p}}$ th belong to the Spectrum leakage values, the sum of all discrete spectral lines should be value 1. And (1- $(y p-1+y p+1))$ can be taken as all leakage lines are superimposed on the line $k_{\mathrm{p}}$ except the line $k_{\mathrm{p}-1}$ and $k_{\mathrm{p}+1}$, results obtained from $\left.1-\left(y_{\mathrm{p}-1}+y_{\mathrm{p}+1}\right)\right) / y_{\mathrm{p}-1}$ or $\left(1-\left(y_{\mathrm{p}-1}+y_{\mathrm{p}+1}\right)\right) / y_{\mathrm{p}+1}$ are given to spectrum line $k_{\mathrm{p}}$ as weighted value, which is accord with the practical situation. In this paper, average value of $\left(1-\left(y_{\mathrm{p}-1}+y_{\mathrm{p}+1}\right)\right) / y_{\mathrm{p}-1}$ and $\left(1-\left(y_{\mathrm{p}-1}+y_{\mathrm{p}+1}\right)\right) / y_{\mathrm{p}+1}$ is taken as weighted value of spectrum line $k_{\mathrm{p}}$, which is about 2.096. Therefore, weighted value of $k_{\mathrm{p}-1}$ th 、 $k_{\mathrm{p}}$ th $、 k_{\mathrm{p}+1}$ th spectrum line are $1 、 2.096 、 1$, respectively. The weighted average value of amplitude is

$$
A_{0}=\frac{R}{\left|W\left(\frac{2 \pi(-\alpha-1)}{N}\right)\right|+2.096\left|W\left(\frac{2 \pi(-\alpha)}{N}\right)\right|+\left|W\left(\frac{2 \pi(-\alpha+1)}{N}\right)\right|}
$$


with $R=A_{\mathrm{p}-1}\left|W\left(\frac{2 \pi(-\alpha-1)}{N}\right)\right|+2.096 A_{\mathrm{p}}\left|W\left(\frac{2 \pi(-\alpha)}{N}\right)\right|+A_{\mathrm{p}+1}\left|W\left(\frac{2 \pi(-\alpha+1)}{N}\right)\right|$.

According to $R=2\left(y_{\mathrm{p}-1}+2.096 y_{\mathrm{p}}+y_{\mathrm{p}+1}\right), \quad$ (7) is deduced as

$$
A_{0}=\frac{2\left(y_{\mathrm{p}-1}+2.096 y_{\mathrm{p}}+y_{\mathrm{p}+1}\right)}{\left|W\left(\frac{2 \pi(-\alpha-1)}{N}\right)\right|+2.096\left|W\left(\frac{2 \pi(-\alpha)}{N}\right)\right|+\left|W\left(\frac{2 \pi(-\alpha+1)}{N}\right)\right|}
$$

When $N$ is very large, (8) is simplified as

$$
A_{0}=N^{-1}\left(y_{\mathrm{p}-1}+2.096 y_{\mathrm{p}}+y_{\mathrm{p}+1}\right) v(\alpha)
$$

Frequency correction is

$f_{0}=\left(\alpha+k_{\mathrm{p}}\right) \Delta f$

By the formula (5), initial phase correction is obtained

$$
\varphi_{0}=\arg [X(k \Delta f)]+\frac{\pi}{2}-\arg \left[W\left(\frac{k \Delta f-f_{0}}{f_{s}}\right)\right]
$$

DFT is used for Blackman-Harris window, which means $w=2 \pi k / N$ is brought into (2), and we obtain

$$
\begin{aligned}
& W\left(\frac{2 \pi k}{N}\right)=0.35875 W_{\mathrm{R}}\left(\frac{2 \pi k}{N}\right)-0.224145\left[W_{\mathrm{R}}\left(\frac{2 \pi k}{N}-\frac{2 \pi}{N}\right)+W_{\mathrm{R}}\left(\frac{2 \pi k}{N}+\frac{2 \pi}{N}\right)\right]+ \\
& 0.07064\left[W_{\mathrm{R}}\left(\frac{2 \pi k}{N}-\frac{4 \pi}{N}\right)+W_{\mathrm{R}}\left(\frac{2 \pi k}{N}+\frac{4 \pi}{N}\right)\right]-0.00584\left[W_{\mathrm{R}}\left(\frac{2 \pi k}{N}-\frac{6 \pi}{N}\right)+W_{\mathrm{R}}\left(\frac{2 \pi k}{N}+\frac{6 \pi}{N}\right)\right]
\end{aligned}
$$

with $W_{\mathrm{R}}\left(\frac{2 \pi k}{N}\right)=\frac{\sin (\pi k)}{\sin (\pi k / N)} e^{-\mathrm{j} \frac{N-1}{N} \pi k}$.

By the formula (5), $\quad k_{\mathrm{p}}$ th spectrum line amplitude $y_{\mathrm{p}}=\left|X\left(k_{\mathrm{p}} \times \Delta f\right)\right|=\left|\left(A_{0} / 2\right) \times W\left[2 \pi\left(k_{\mathrm{p}}-k_{0}\right) / N\right]\right|$, to make a transform $\alpha=k_{0}-k_{\mathrm{p}}$, and we have $k_{\mathrm{p}}-k_{0}=-\alpha$ and bring it into (12), $\left|W\left(2 \pi\left(k_{\mathrm{p}}-k_{0}\right) / N\right)\right|=|W(2 \pi(-\alpha) / N)|$ is obtained, then we can obtain $\left|W\left(2 \pi\left(k_{\mathrm{p}-1}-k_{0}\right) / N\right)\right|$ (namely $|W(2 \pi(-\alpha-1) / N)|)$ and $\left|W\left(2 \pi\left(k_{\mathrm{p}+1}-k_{0}\right) / N\right)\right|$ (namely $\left.|W(2 \pi(-\alpha+1) / N)|\right)$ in the same way, and bring them into (6). Using polyfit $\left(^{*}\right)$ function to carry out polynomial fitting and we can obtain approximate expression $\alpha=F(\beta)$ of $\alpha=f^{1}(\beta)$, by the same way, $\left|W\left(2 \pi\left(k_{\mathrm{p}}-k_{0}\right) / N\right)\right| 、\left|W\left(2 \pi\left(k_{\mathrm{p}-1}-k_{0}\right) / N\right)\right|$ and $\left|W\left(2 \pi\left(k_{\mathrm{p}+1}-k_{0}\right) / N\right)\right|$ are brought into (8), and approximate expression of (9) is obtained.

$$
A_{0}=N^{-1}\left(y_{\mathrm{p}-1}+2.096 y_{\mathrm{p}}+y_{\mathrm{p}+1}\right) g(\alpha)
$$

Take a set of values $\alpha$ in [-0.5, 0.5], corresponding value $\beta$ and $v(\alpha)$ are obtained from (6) and (9). Call polyfit $(\beta, \alpha, m)$ function ( $m$ is the order of fitting polynomial) to inverse fit and obtain coefficients of the polynomial $F(\beta)$. The coefficients of the polynomial $g(\alpha)$ are obtained by calling polyfit $(\alpha, v(\alpha), m)$ function. Finally, correction formula for interpolation of three spectral lines based on Blackman-Harris window is obtained

$$
\alpha=0.93891885 \beta-0.082038310 \beta^{3}+0.01541147 \beta^{5}-0.00316988 \beta^{7}
$$

And amplitude correction formula is

$$
A_{0}=\left(y_{\mathrm{p}-1}+2.096 y_{\mathrm{p}}+y_{\mathrm{p}+1}\right) \times\left(1.6126041021+0.44103999 \alpha^{2}+\right.
$$




$$
\begin{aligned}
& \left.0.06420677 \alpha^{4}+0.0069132572 \alpha^{6}\right) / N \\
\varphi_{0}= & \arg \left[X\left(k_{\mathrm{p}} \Delta f\right)\right]+\frac{\pi}{2}-\alpha \pi
\end{aligned}
$$

\section{Simulation experiment analysis}

In order to verify the effectiveness of the proposed algorithm, the harmonic components with multiple frequencies are designed, and the amplitude, frequency and phase of the fundamental and harmonics are shown in Table 2.

Table 2. The amplitude, frequency and phase of simulated harmonic signal

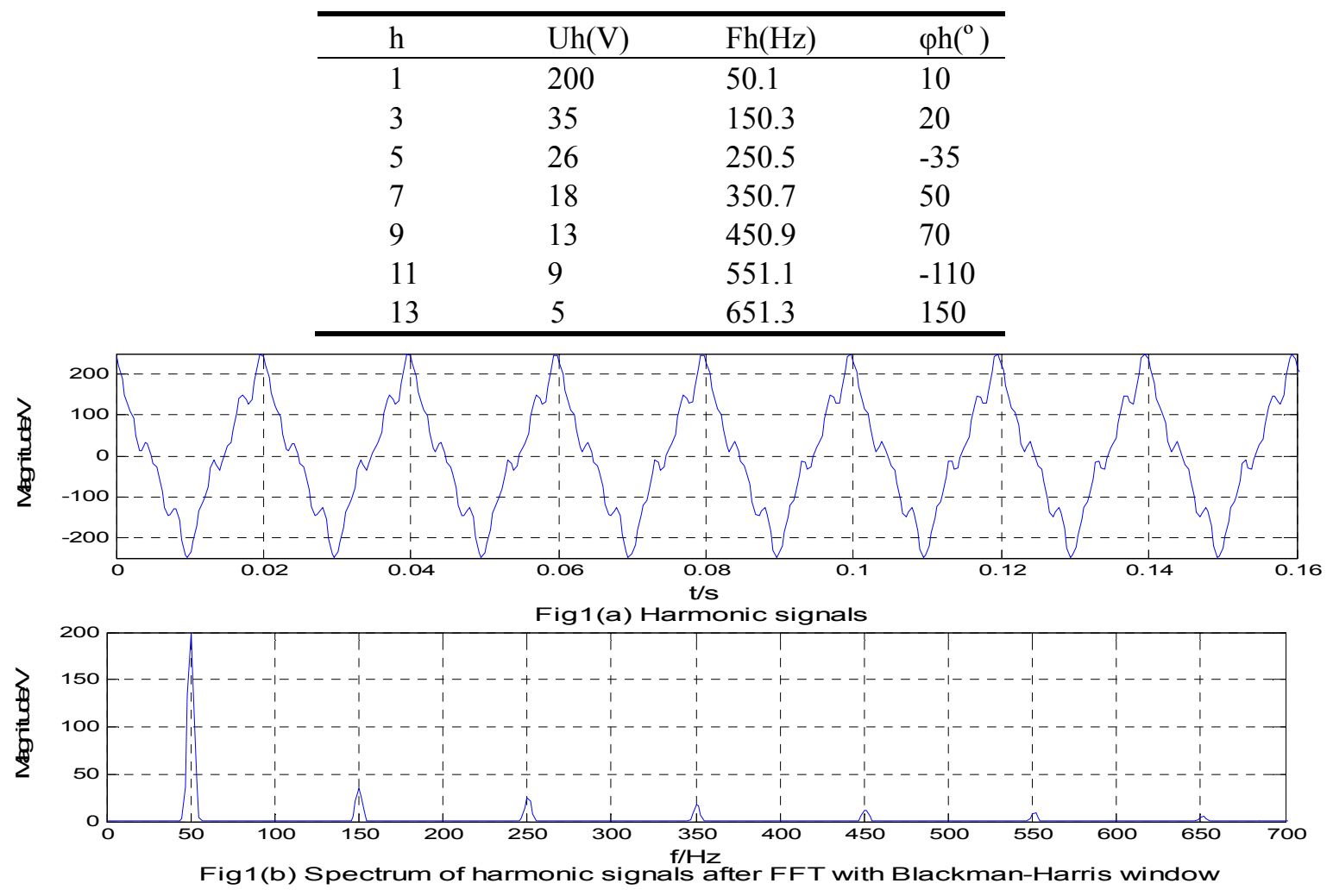

Figure 1. Harmonic signals and the spectrum

Compared with the Ref. [10], this paper gives different weighted values of the $k_{\mathrm{p}}$ th spectrum line during the derivation of amplitude correction formula (15), which is different from Ref. [10]. The formula of the parameter $\alpha$ is same as that of Ref. [10]. Therefore, the amplitude parameters of this algorithm are different from Ref. [10], while the frequency and phase parameters are basically the same. Simulation experiment is carried out by taking proposed algorithm as an example to analyze frequency and phase of the harmonic wave, and simulation results are compared with Blackman

Table 3. Amplitude analysis results of different algorithms

\begin{tabular}{lccccc}
\hline & $\begin{array}{c}\text { Theoretical } \\
\text { Value }\end{array}$ & $\begin{array}{c}\text { Blackman } \\
\text { Double } \\
\text { Interpolation FFT }\end{array}$ & $\begin{array}{c}\text { Blackman-Harris } \\
\text { Double Interpolation } \\
\text { FFT }\end{array}$ & $\begin{array}{c}\text { Common } \\
\text { Triple-spectrum-line } \\
\text { Interpolation FFT }\end{array}$ & $\begin{array}{c}\text { Proposed } \\
\text { Algorithm }\end{array}$ \\
\hline 1 & 200 & 199.9996982 & 200.0000170 & 199.9999986 & 199.99999995 \\
3 & 35 & 35.0000721 & 35.0000232 & 34.99999997 & 35.00000001 \\
5 & 26 & 26.0000756 & 26.0000102 & 25.9999992 & 26.0000003 \\
7 & 18 & 17.9999466 & 17.9999894 & 18.0000006 & 18.0000001 \\
9 & 13 & 13.0001214 & 12.9999982 & 12.9999999 & 12.9999999 \\
11 & 9 & 8.9999288 & 9.0000037 & 8.9999995 & 8.9999997 \\
13 & 5 & 4.9999949 & 5.0000009 & 4.9999999 & 5.0000000 \\
\hline
\end{tabular}


window and Blackman-Harris window double-spectrum-line interpolation FFT algorithm. When the amplitude harmonic signals are analyzed, proposed correction formula should be compared with Blackman-Harris window triplet correction formula (hereafter refer to as the conventional three-line algorithm) given by Ref. [10]. Sampling frequency equals $2560 \mathrm{~Hz}$, sampling points are 512, simulation and data analysis results are shown in Figure 1 to 4, and Table 3 to Table 5.

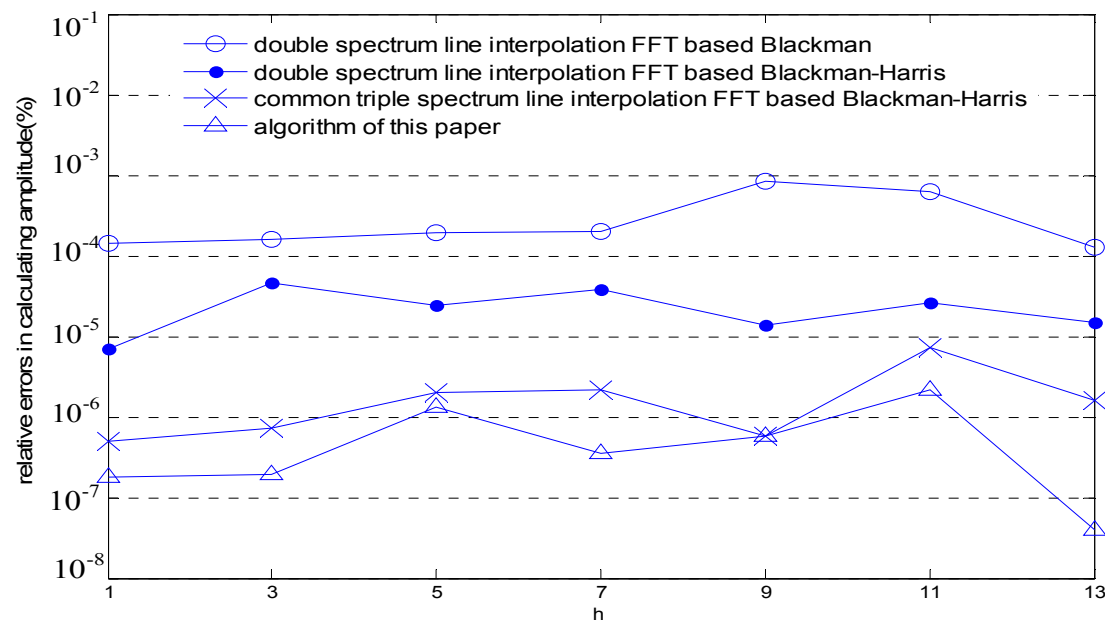

Figure 2. Relative errors of amplitude

Figure 1 (a) is a signal waveform containing a plurality of harmonic frequency components, fundamental waveform distortion occurs due to the existence of 3 times, 5 times $\sim 13$ times harmonics. Figure 1 (b) is frequency spectrogram which is obtained from the signal processed by Blackman-Harris window and FFT afterwards. The figure shows that proposed algorithm can analyze the frequency and amplitude of the fundamental and harmonic. Tables 3, 4 and 5 are specific results calculated by different algorithm, containing amplitude, frequency and phase of fundamental and each harmonic signal. Figure 2, 3 and 4 are relative errors of amplitude, frequency and phase of different algorithms, respectively. The figures show that, compared with the Blackman window double spectrum line interpolation FFT algorithm, Blackman-Harris window double spectrum line interpolation FFT algorithm is more accurate, for sidelobe peak level of Blackman-Harris window is far below that of Blackman window, reducing the infection in spectral leakage. Proposed algorithm in this paper has a higher computational accuracy compared with the Blackman-Harris window double spectrum line interpolation FFT algorithm, which shows triple-spectrum-line interpolation algorithm make fully use of three spectrum lines near the harmonic frequency point to carry out FFT analysis and its correction calculation results have a higher accuracy than that of doublet interpolation FFT algorithm. From table 3 and figure 3, different weighted value are given to deduce three spectral lines interpolation amplitude correction formula, relative error of the amplitude parameters calculated in this paper is sightly below the result calculated by amplitude correction formula given in Ref. [10], the accuracy and effectiveness of the proposed algorithm are verified.

Table 4. Frequency analysis results of different algorithms

\begin{tabular}{|c|c|c|c|c|}
\hline & $\begin{array}{c}\text { Theoretical } \\
\text { Value }\end{array}$ & $\begin{array}{c}\text { Blackman } \\
\text { Double } \\
\text { Interpolation FFT }\end{array}$ & $\begin{array}{c}\text { Blackman-Harris } \\
\text { Double } \\
\text { Interpolation FFT }\end{array}$ & $\begin{array}{l}\text { Proposed } \\
\text { Algorithm }\end{array}$ \\
\hline 1 & 50.1 & 50.1000197 & 50.0999984 & 50.0999999 \\
\hline 3 & 150.3 & 150.2999815 & 150.2999897 & 150.3000011 \\
\hline 5 & 250.5 & 250.4999577 & 250.4999927 & 250.5000002 \\
\hline 7 & 350.7 & 350.7000599 & 350.7000137 & 350.7000014 \\
\hline 9 & 450.9 & 450.8998162 & 450.9000029 & 450.9000005 \\
\hline 11 & 551.1 & 551.1001517 & 551.0999848 & 551.0999969 \\
\hline 13 & 651.3 & 651.3000248 & 651.2999946 & 651.2999996 \\
\hline
\end{tabular}




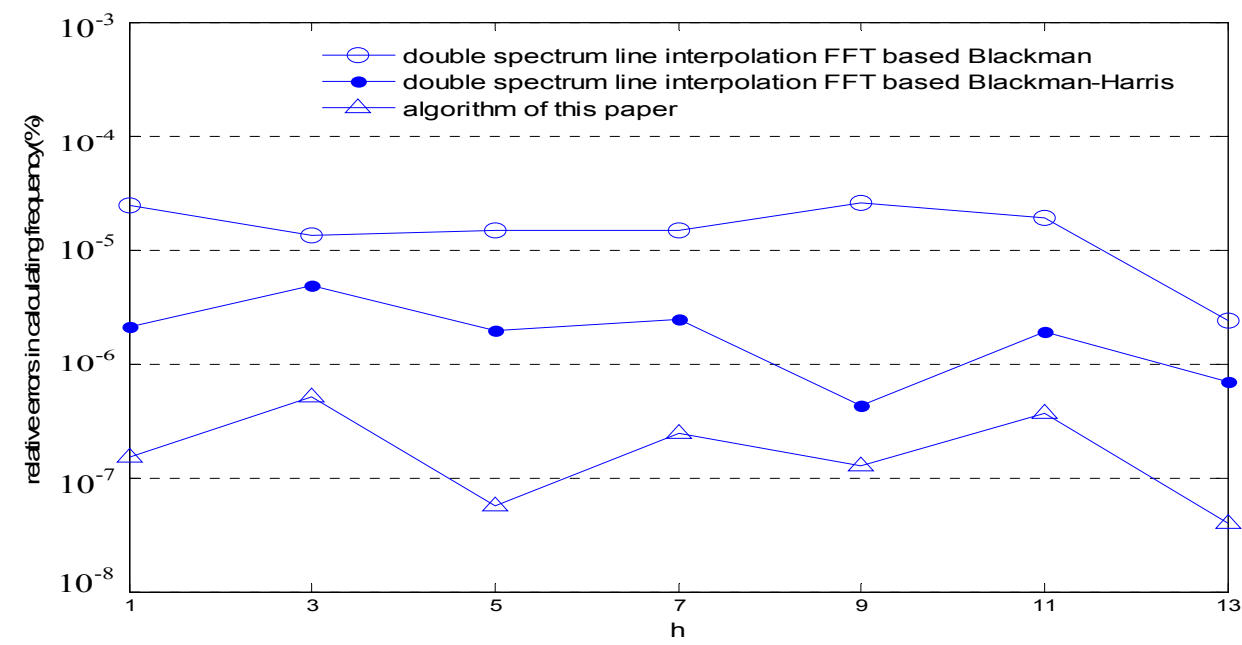

Figure 3. Relative errors of frequency

Table 5. Amplitude analysis results of different algorithms

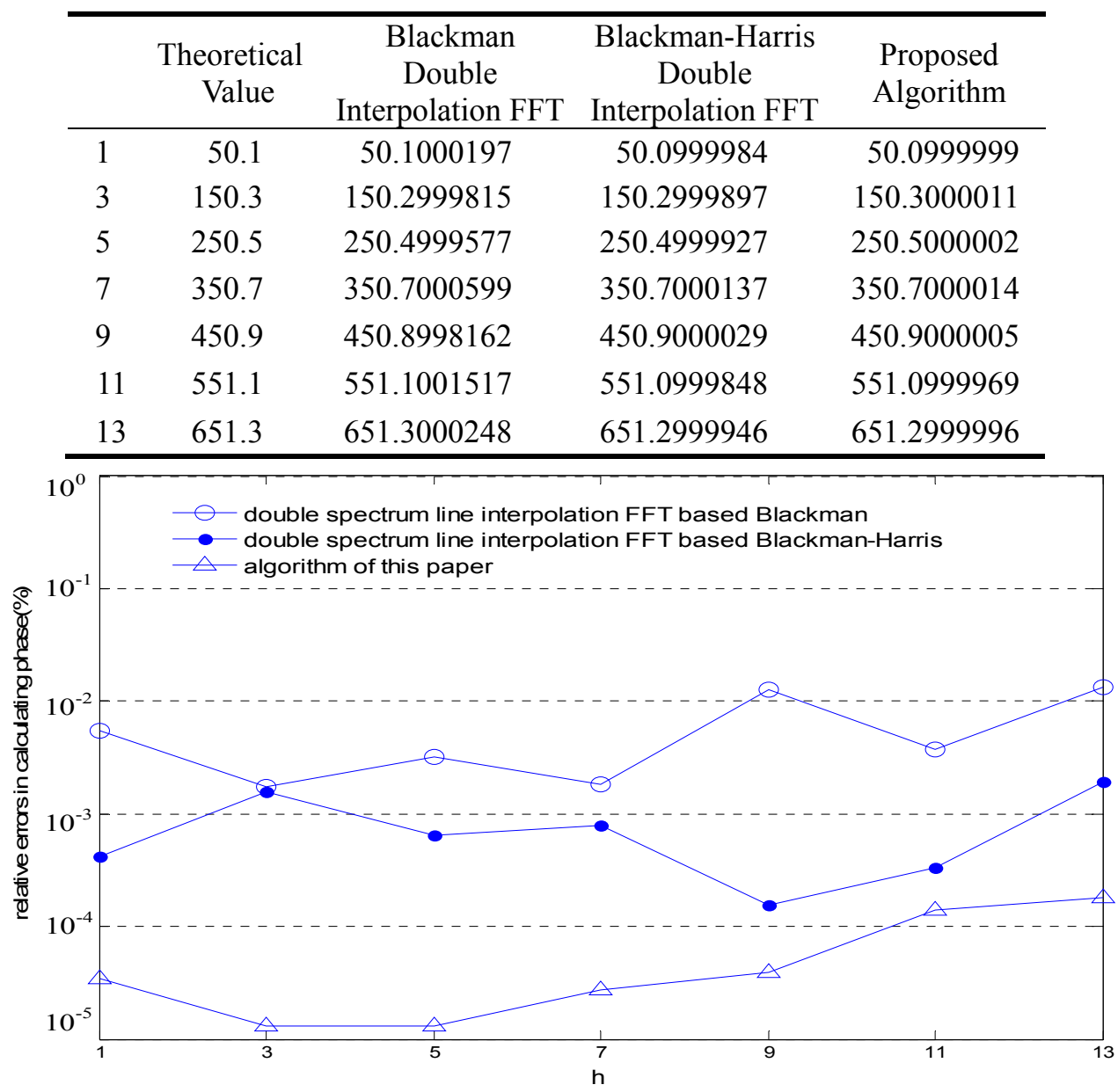

Figure 4. Relative errors of phase

\section{Conclusion}

In this paper, spectral characteristics of Blackman-Harris window is analyzed. Results show that the peak sidelobe level of the Blackman-Harris window is low. With this special spectral characteristic, spectrum leakage effect will be effectively reduced. Triple-spectrum-line interpolation FFT Algorithm based on Blackman-Harris window is proposed. Using three spectrum lines near the harmonic frequency point and curve fitting function, we can deduce practical 
correction formula of frequency and phase. Leakage spectral lines outside of three spectral lines are superimposed on the maximum amplitude spectrum near harmonic frequency point, and we take the averages of superimposed results and the ratio of two adjacent spectral lines as weighted values, deducing the amplitude correction formula. Matlab simulation results show that, compared with doublet interpolation FFT algorithm based on Blackman-Harris window or Blackman window, algorithm proposed in this paper has a higher accuracy of harmonic parametric analysis. Compared with the amplitude correction formula of three spectral lines given by [10], the relative error of the algorithm in this paper is lower, and the accuracy is significantly improved.

\section{Acknowledgement}

This research was financially supported by the Natural Science Fund of Hubei Province (grant number 2015CFB219).

\section{References}

[1] ZHOU Xi-feng, ZHAO Rong, GUO Qian-gang, Blackman-Harris Window Based Interpolation FFT Harmonic Analysis and Its Application[J], Electrical Measurement \& Instrumentation. 2014, 11: 1001-1390.

[2] Wen He, Teng Zhaosheng, Qing Baiyuan. Hanning Self-Convolution Windows and Its Application to Harmonic Analysis[J]. Transactions of China Electrotechnical Socirty, 2009, 24(2): 164-169.

[3] HAO QIAN, RONGXIANG ZHAO, TONG CHEN. Interharmonics analysis based on interpolating windowed FFT algorithm[J]. IEEE Transactions on Power Delivery, 2007, 22(2): 1064-1069.

[4] Wen He, Teng Zhaosheng, Wang Yong. Improved Windowed Interpolation FFT Algorithm and Application for Power Harmonic Analysis [J]. Transactions of China Electrotechnical Socirty, 2012, 27(12): 270-277.

[5] ZHANG Xue-wu, YAN Ping. Harmonics Analysis and Simulation Study on Power System[J]. Computer Simulation, 2005, 22(9): 195-200.

[6] ZHOU Jun, Wang Xiao-hai, Qi Cai-jun. Estimation of electrical harmonic parameters by using the Interpolated FFT algorithm based on Blackman window[J]. IEEE Transactions on Power Delivery, 2007, 22(2): 1064-1069.

[7] QING Bai-yuan, TENG Zhao-sheng, GAO Yun-peng. An Approach for Electrical Harmonic Analysis Based on Nuttall Window Double-spectrum-line Interpolation FFT [J]. CHINESE SOCIETY FOR ELECTRICAL ENGINEERING, 2008, 28(25): 153-158.

[8] JIANG Chun-fang, LIU Min. Inter-harmonics analysis based on double interpolation FFT algorithm[J]. Power System Protection and Control, 2010,38(3):11-14.

[9] WANG Liu-wang, HUANG Jian-cai, SUN Jian-xin. An improved precise algorithm for harmonic analysis based on Hanning-windowed FFT[J]. Power System Protection and Control, 2012, 40(24): 28-33.

[10] NIU Shengsuo, LIANG Zhirui, ZHANG Jianhua. An Algorithm for Electrical Harmonic Analysis Based on Triple-spectrum-line Interpolation FFT[J]. CHINESE SOCIETY FOR ELECTRICAL ENGINEERING, 2012, 32(16): 130-135. 